\title{
Adherencia a la dieta sin gluten y calidad de vida en relación con la salud en los celiacos del Paraguay, 2021
}

DOI: 10.17533/udea.penh.v23n2a04

PERSPECTIVAS EN NUTRICIÓN HUMANA

ISSN 0124-4108

Escuela de Nutrición y Dietética, Universidad de Antioquia. Medellín, Colombia

Vol. 23, N. ${ }^{\circ}$ 2, julio-diciembre de 2021, pp. 171-182.
Artículo recibido: 25 de mayo de 2021

Aprobado: 25 de noviembre de 2021

\section{Raúl Emilio Real-Delor ${ }^{1 *}$; María Elena Chamorro-Aguilera²}

\section{- - Resumen}

Antecedentes: la calidad de vida en relación con la salud de las personas con enfermedad celiaca puede estar influenciada por la adherencia a la dieta sin gluten. Objetivos: describir las características demográficas y clínicas de los celiacos, evaluar la adherencia dietética con el cuestionario CDAT de Leffler y medir la calidad de vida autopercibida utilizando el cuestionario CD-QOL. Métodos y materiales: diseño observacional, transversal y prospectivo. Se aplicó una encuesta telemática a adolescentes y adultos de Paraguay diagnosticados con enfermedad celiaca, entre febrero y abril del año 2021, que accedieron a participar del estudio. La adherencia dietética se determinó con el cuestionario Celiac Dietary Adherence Test de Leffler y la calidad de vida con el CD-QOL de Dorn. Se midieron además variables demográficas y clínicas. La investigación fue aprobada por el Comité de Ética de la Universidad Privada del Este, en Paraguay. Resultados: la muestra estuvo constituida por 344 sujetos de estudio, de los cuales el $87 \%$ pertenecía al sexo femenino. La edad media de los adolescentes fue de 15,1 años, y la de los adultos de $38 \pm 13$ años. La adherencia a la dieta sin gluten se encontró en el $78 \%(n=268)$ de los encuestados; fue buena en el $59 \%$, moderada en el $19 \%$ y mala en el $22 \%$ de los encuestados. La calidad de vida fue buena en el $30 \%(n=103)$ de los sujetos y mala en el $70 \%(n=241)$. Se encontró asociación entre los sujetos sin o mala adherencia a la dieta con la mala calidad de vida $(p<0,006)$. Conclusión: en los celiacos estudiados, la mala adherencia a la dieta sin gluten se asocia con menor calidad de vida en relación con la salud.

Palabras clave: enfermedad celiaca, dieta sin gluten, calidad de vida, cumplimiento y adherencia al tratamiento, Paraguay.

$1^{*}$ Autor de correspondencia. Doctor en Medicina, Universidad Privada del Este, Asunción, Paraguay. raulemilioreal@gmail.com. https://orcid.org/0000-0002-5288-5854

2 Bioquímica, Fundación Paraguaya de Celiacos. Paraguay. mariaelenaagui @ hotmail.com. https://orcid.org/0000-0003-1875-6829

Cómo citar este artículo: Real-Delor RE, Chamorro-Aguilera ME. Adherencia a la dieta sin gluten y calidad de vida en relación con la salud en los celiacos del Paraguay, 2021. Perspect Nutr Humana. 2021;23:171-82. DOI: 10.17533/udea.penh.v23n2a04 


\section{Adherence to a Gluten-Free Diet and Health-Related Quality of Life in Celiac Patients from Paraguay, 2021}

\section{Abstract}

Background: The health-related quality of life of people with celiac disease may be influenced by adherence to a gluten-free diet. Objectives: To describe the demographic and clinical characteristics of celiac patients, to evaluate dietary adherence with the Leffler CDAT questionnaire, and to measure self-perceived quality of life using the CD-QOL questionnaire. Material and Methods: Observational, cross-sectional, and prospective design. A telematic survey was applied to adolescents and adults in Paraguay, diagnosed with celiac disease, between February and April 2021, who agreed to participate in the study. Dietary adherence was determined with the Celiac Dietary Adherence Test of Leffler and quality of life with the test CD-QOL of Dorn. Demographic and clinical variables were also measured. The research was approved by the Ethics Committee of the Universidad Privada del Este, in Paraguay. Results: The sample consisted of 344 study subjects of which $87 \%$ belonged to the female sex. The mean age of the adolescents was $15 \pm 1$ years, and that of the adults was $38 \pm 13$ years. Adherence to a gluten-free diet was found in $78 \%(n=268)$ of those surveyed, it was good in $59 \%$, moderate in $19 \%$, and bad in $22 \%$. The quality of life was good in $30 \%(n=103)$ of the subjects and bad in 70\% (n 241). It was found an association between subjects with no or bad adherence to the diet with bad quality of life $(p<0.006)$.. Conclusion: In the celiac patients studied, poor adherence to the gluten-free diet is associated with worse health-related quality of life.

Keywords: Celiac disease, gluten-free diet, quality of life, compliance and adherence to treatment, Paraguay.

\section{INTRODUCCIÓN}

La enfermedad celiaca es una afección de origen autoinmune, crónica, sistémica, producida por la ingestión de gluten en personas genéticamente predispuestas (1). Su tratamiento se basa principalmente en una dieta sin gluten (DSG) estricta y continua, que requiere una formación, motivación y seguimiento significativos del paciente (2). La ingesta continua de gluten genera complicaciones crónicas y la probabilidad de desarrollar neoplasias del tubo digestivo (3).

En esta investigación se definió la calidad de vida en relación con la salud y la percepción que tiene el individuo de los aspectos de su salud general: cómo se siente en relación con la enfermedad misma y los efectos que tienen los tratamientos médicos sobre las personas (4). La DSG puede afectar favorable o desfavorablemente la calidad de vida de las personas con enfermedad celiaca.
Por un lado, permite mejorar los síntomas, pero, por otro, desmejora la calidad de vida previa del que la padece $(5,6)$.

Para medir la calidad de vida existen cuestionarios en español como el CD-QOL (7). En 2017, en las personas celiacas de Paraguay se encontró que la calidad de vida autopercibida era regular en el 49 , mala en el 26 y buena en solo el $25 \%$ de las personas, pero dicho estudio no determinó la asociación con la DSG usando instrumentos validados (4). Revisiones sistemáticas revelan que la estricta adherencia a la DSG mejora la calidad de vida, sobre todo en aquellas personas que presentaban molestias digestivas al momento del diagnóstico $(3,8)$. La adherencia a la DSG puede ser medida por determinaciones de laboratorio de los autoanticuerpos (transglutaminasa tisular $\lg \mathrm{A}$ ), biopsias duodenales, entrevistas con nutricionistas y con encuestas de autorreporte. Las encuestas son métodos indirectos válidos 
para medir la adherencia a la DSG (9); es el caso del cuestionario Celiac Dietary Adherence Test (CDAT) de Leffler, que ha demostrado elevada sensibilidad y especificidad para detectar este fenómeno (10).

Cada sociedad o grupo de individuos puede presentar diversas facilidades y dificultades para realizar la DSG. Existen factores individuales y comunitarios que deben identificarse, pues tienen impacto para la adherencia a esta dieta (1). Existe limitada evidencia sobre la naturaleza y la magnitud de los factores relacionados a la adherencia a la DSG y su relación con la calidad de vida, sobre todo en Paraguay (4). Los problemas del día a día con la DSG y el estilo de vida en los celiacos de este país no pueden extrapolarse de otras investigaciones, por lo que se requieren datos nacionales (11).

Los objetivos de esta investigación fueron tres: describir las características demográficas y clínicas de los celiacos, evaluar la adherencia dietética con el cuestionario CDAT de Leffler y medir la calidad de vida autopercibida utilizando el cuestionario CD-QOL.

\section{MATERIALES Y MÉTODOS}

\section{Diseño}

Observacional, transversal. La población de estudio la constituyeron hombres y mujeres, mayores de 14 años de edad, con enfermedad celiaca, que vivían en el Paraguay en el periodo enero-abril 2021 y que aceptaron completar una encuesta. Para asegurar que los sujetos del estudio fueran celiacos confirmados se realizó la invitación para participar de la encuesta utilizando los datos de los archivos de la Fundación Paraguaya de Celiacos, en la que se registra solo a aquellos con confirmación histológica. Se excluyeron los cuestionarios incompletos. Se utilizó un muestreo no probabilístico, por conveniencia.

\section{Variables}

Se midieron variables demográficas (edad, sexo, ciudad de residencia, independencia económica, nivel académico) y clínicas (peso, talla, edad del diagnóstico confirmatorio de la enfermedad celiaca, cuadro clínico inicial, síntomas por trasgresión alimentaria al gluten, comorbilidades, antecedentes falmiliares). La adherencia a la DSG se consideró como variable independiente y la calidad de vida como dependiente. Se consideraron adolescentes a aquellos sujetos con edades entre los 13 y 17 años (12).

\section{Instrumentos de medición}

La prueba CDAT de Leffler consta de siete preguntas, esta se midió como variable ordinal con una escala Likert que va del 1 al 5 , otorgándose un punto a la opción más baja y cinco puntos a la más alta. Esta prueba generó valores totales entre 7 y 35 puntos. Se consideró buena adherencia a la DSG a todo valor $\leq 13$ puntos, moderada si estaba entre 14 y 16 puntos y mala si era $\geq 17$ puntos (8,13-15). Con fines estadísticos, se consideraron dos grupos: aquellos con mala adherencia (puntaciones superiores a 17) y los que tenían buena y moderada adherencia a la DSG (puntuaciones inferiores a 16).

La calidad de vida se midió con el cuestionario CD-QOL. Este instrumento consiste en 20 preguntas cuyas respuestas se puntúan con una escala de Likert del 1 al 5 . La sumatoria final varía desde 0 puntos (peor calidad de vida) hasta 100 (mejor calidad de vida) excepto el ítem 8 , que se puntúa a la inversa $(8,12)$. Las preguntas se refieren a cuatro dimensiones: disforia, limitaciones, salud y tratamiento inadecuado. Aplicando el punto de 
corte 70 se dividió la muestra en buena $(p \geq 70)$ y mala calidad de vida $(p \leq 69)(5,7)$.

Ambos cuestionarios estaban disponibles en español y fueron validados a partir de una prueba piloto con 30 muestras. La consistencia interna se determinó con el alfa de Cronbach utilizando el programa Epi Dat 3.1 $1^{\mathrm{TM}}$. Se obtuvo un coeficiente 0,78 para la adherencia a la DSG y 0,87 para la calidad de vida.

\section{Gestión de datos}

La encuesta, generada por los autores y divulgada a través de las redes sociales y las filiales de la Fundación Paraguaya de Celiacos en todo el país, se aplicó en internet por medio de la plataforma virtual Google Forms ${ }^{\mathrm{TM}}$.

\section{Tamaño de la muestra}

Se calculó con el programa Epi Dat 3.1 ${ }^{\mathrm{TM}}$. Según estudios previos, se esperaba una frecuencia del $25 \%$ de sujetos con una buena calidad de vida (4). Utilizando un nivel de confianza del $95 \%$ y de precisión del $5 \%$, se calculó incluir al menos 289 encuestados. Esta muestra se obtuvo de un registro de 1200 celiacos confirmados a la fecha por la Fundación Paraguaya de Celiacos.

\section{Análisis estadístico}

Las respuestas fueron registradas en una planilla electrónica y sometidas a estadística descriptiva con el programa Epi Info $7^{\mathrm{TM}}$. Las variables cualitativas se expresaron en frecuencias y porcentajes, mientras que las cuantitativas se resumieron en medidas de tendencia central y de dispersión. Posteriormente, se aplicaron pruebas estadísticas reagrupando a los sujetos según su calidad de vida y adherencia a la dieta. Se utilizaron la prueba ji al cuadrado, la prueba exacta de Fischer, la prueba de Kruskal-Wallis y la prueba $t$ de Student para evaluar la asociación entre variables. Esta asociación se consideró significativa si $p<0,05$. La hipótesis fue que en esta muestra la adherencia a la DSG se asociaba con mejor calidad de vida en relación con la salud.

\section{Consideraciones éticas}

Se respetaron los principios de la bioética. Se guardó la confidencialidad de los datos personales, pues la encuesta era anónima. Antes de diligenciarla, se explicaron los motivos del estudio, el responsable del manejo de los datos y un consentimiento para participar de la encuesta. El protocolo fue aprobado por el Comité de Ética de la Facultad de Medicina de la Universidad Privada del Este, Paraguay.

\section{RESULTADOS}

En la figura 1 se muestra el flujograma de inclusión y resultados de la calidad de vida. Se evidencia que, de un total de 354 personas a quienes se les aplicó la encuesta, se excluyeron diez, por falta de datos. De las 344 encuestas analizadas, el 78 \% (n 268) mostró adherencia a la DSG y de estas el 34 \% (n 91) mostró una percepción de buena calidad de vida.

En cuanto a las características demográficas, se encontró que predominó el sexo femenino (87\%), la mayoría eran adultos $(93,9 \%)$ con edad media de $38 \pm 13$ años. La edad media de los adolescentes (n 21) fue de $15 \pm 1$ año. Respecto a la procedencia, principalmente provenían de Asunción ( $47 \%$ ) y el nivel educativo predominante fue universitario (78\%), además se encontró que la mayoría $(70 \%)$ contaba con ingresos propios (Tabla 1). La mediana de la edad al momento del diagnóstico fue de 28 años, con rango de 19 a 37 años. La mediana de la duración de la dieta sin gluten al momento de la encuesta fue de 8 años, con rango de 3 a 13 años. 


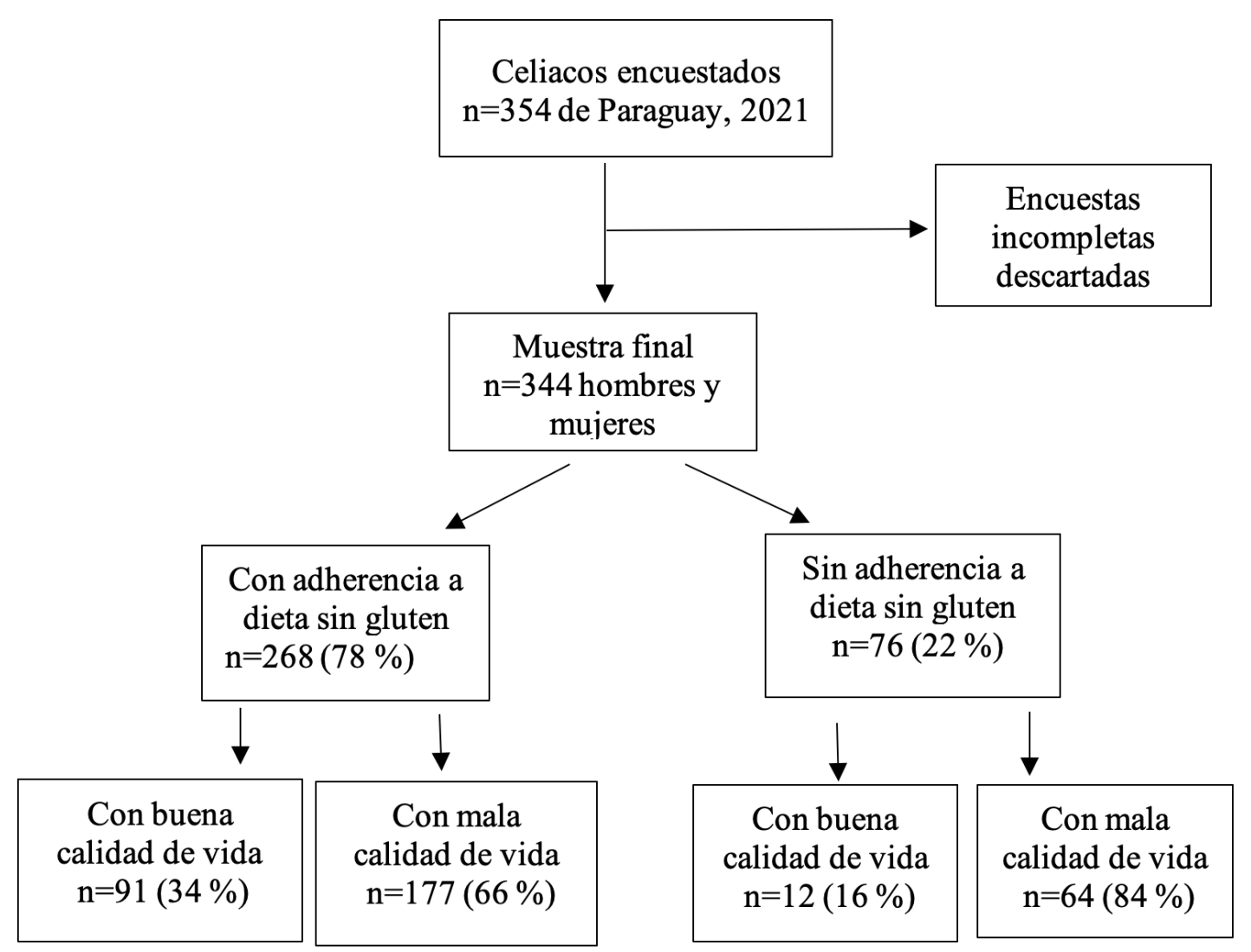

Figura 1. Flujograma de inclusión y resultados. La muestra final del estudio fue de 344 sujetos, de los cuales 268 tenían adherencia a la DSG y 76 no la tenían.

Tabla 1. Características demográficas de los celiacos de Paraguay encuestados, 2021

\begin{tabular}{llcc}
\hline \multirow{2}{*}{ Características demográficas } & \multicolumn{2}{c}{$\begin{array}{c}\text { Total } \\
\text { Sexo }\end{array}$} & \multicolumn{2}{c}{$(\mathbf{n}=344)$} \\
\cline { 2 - 4 } & Femenino & $\%$ \\
\hline \multirow{2}{*}{ Procedencia } & Masculino & 299 & 86,9 \\
& Asunción & 45 & 13,1 \\
& Departamento central & 106 & 30,8 \\
Nivel educativo & Otros departamentos & 75 & 21,4 \\
& Primaria & 8 & 2,3 \\
Independencia & Secundaria & 68 & 19,8 \\
económica & Universitaria & 268 & 77,9 \\
& Con ingresos propios & 242 & 70,3 \\
& Sin ingresos propios & 102 & 29,7 \\
\hline
\end{tabular}

En cuanto al estado nutricional de los participantes, predominó el exceso de peso. El $37 \%$ tenía sobrepeso y el $10 \%$ obesidad, y en lo que respecta a las características del cuadro clínico, el síntoma predominante fue la diarrea crónica $(84 \%)$. Prácticamente la mitad de los sujetos tenía antecedentes familiares de celiaquía, y en lo que respecta a las comorbilidades, la principal encontrada fue el hipotiroidismo primario (15\%), seguido por la hipertensión arterial (10\%) (Tabla 2). También se encontró que la mediana (rango intercuartílico) de la edad del diagnóstico fue de 28 años (19-37 años) y la mediana de la duración de la dieta sin gluten fue de 8 años (3-13 años). 
Tabla 2. Características clínicas de los celiacos de Paraguay encuestados, 2021

\begin{tabular}{llcc}
\hline \multirow{2}{*}{ Características clínicas } & \multicolumn{2}{c}{ Total } \\
& & \multicolumn{2}{c}{$(\mathbf{n}=344)$} \\
\cline { 2 - 3 } Estado nutricional & Desnutrición & $\%$ \\
\hline & IMC normal & 13 & 3,8 \\
& Sobrepeso & 170 & 49,4 \\
& Obesidad & 128 & 37,2 \\
Cuadro clínico de & 33 & 9,6 \\
debut & Diarrea crónica & 289 & 84,0 \\
& Pérdida de peso & 16 & 4,6 \\
& Anemia & 15 & 4,4 \\
Familiares & Dermatitis herpetiforme & 13 & 3,8 \\
consanguíneos con & Con familiares celiacos & 170 & 3,2 \\
celiaquía & Sin familiares celiacos & 174 & 50,6 \\
& Hipotiroidismo primario & 50 & 14,5 \\
Comorbilidades & Hipertensión arterial & 34 & 9,9 \\
& Diabetes mellitus & 11 & 3,2 \\
& Ninguna & 249 & 72,4 \\
\hline
\end{tabular}

En cuanto a la adherencia a la DSG, se encontró en 268 de los encuestados (78\%), y fue buena en 204 (59\%), moderada en 64 (19\%) y mala en 76 de ellos (22\%). La calidad de vida fue buena en 103 sujetos (30\%) y mala en 241 (70\%). Al describir la frecuencia de la adherencia a la DSG en función de la calidad de vida en relación con la salud, se halló que el porcentaje de sujetos con mala calidad de vida fue más elevado en aquellos sin adherencia a la DSG (84,2\%) en comparación con quienes tenían adherencia moderada $(70,3 \%)$ o buena $(64,7 \%)(p=0,006)$ (Figura 2).

La mediana (rango intercuartílico) de la edad actual fue de 40 (30-52) años en los celiacos con buena calidad de vida y de 34 (25-43) años en aquellos con mala calidad de vida $(p<0,0003$ prueba Kruskal-Wallis). El tiempo en DSG fue de $11 \pm 8$ años en los celiacos con buena calidad de vida y de $9 \pm 8$ años en aquellos con mala calidad de vida con diferencias significativas según la prueba $t$ de Student $(p<0,04)$.

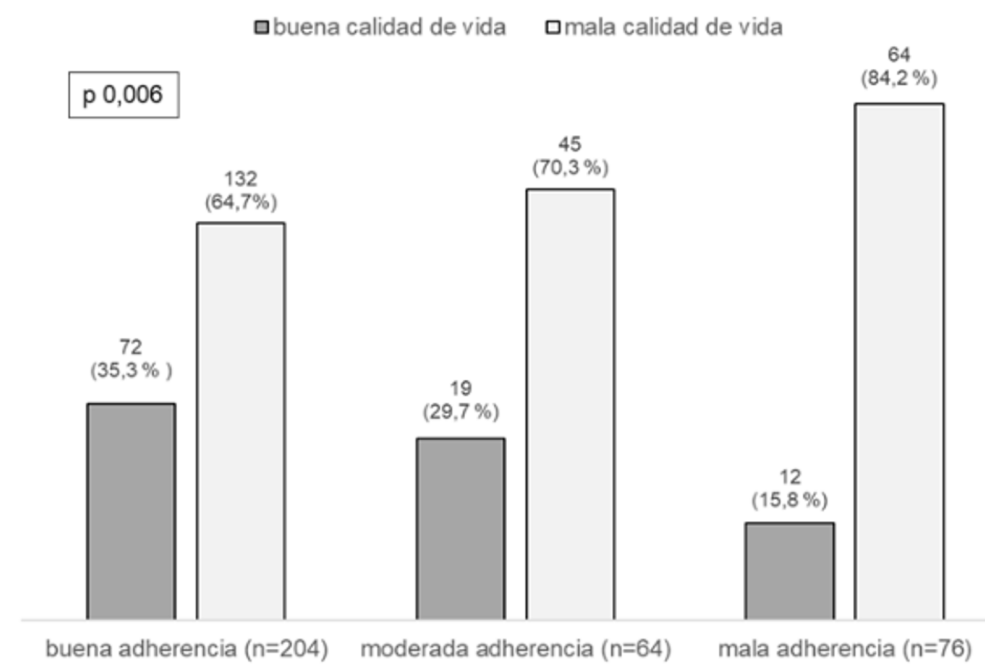

Figura 2. Frecuencia de adherencia a la dieta sin gluten en celiacos con buena y mala calidad de vida.

Tuvieron buena adherencia a la DSG 204 personas, moderada 64 y mala 76. El porcentaje de sujetos con mala calidad de vida fue significativamente mayor entre quienes tuvieron mala adherencia a la DSG, según la prueba ji al cuadrado $(p=0,006)$. 
Otros factores frecuentes en los sujetos con mala calidad de vida fueron el sexo femenino y presentar molestias digestivas al trasgredir la DSG (Tabla 3).
La forma de presentación clínica, sintomática versus asintomática, se distribuyó de igual forma entre los pacientes con buena calidad de vida en relación con la salud.

Tabla 3. Frecuencia de variables demográficas y clínicas en los celiacos con buena y mala calidad de vida

\begin{tabular}{|c|c|c|c|c|c|c|c|c|}
\hline \multirow{3}{*}{ Variables demográficas y clínicas } & & \multicolumn{4}{|c|}{ Calidad de vida } & \multicolumn{2}{|c|}{ Total } & \multirow[t]{2}{*}{ Valor de $\mathrm{p}^{*}$} \\
\hline & & \multicolumn{2}{|c|}{$\begin{array}{l}\text { Buena } \\
(n=103)\end{array}$} & \multicolumn{2}{|c|}{$\begin{array}{c}\text { Mala } \\
(\mathrm{n}=241)\end{array}$} & \multirow[b]{2}{*}{$\mathrm{n}$} & \multirow[b]{2}{*}{$\%$} & \\
\hline & & $\mathrm{n}$ & $\%$ & $\mathrm{n}$ & $\%$ & & & \\
\hline \multirow{2}{*}{ Sexo } & Masculino & 21 & 47 & 24 & 53 & 45 & 100 & \multirow{2}{*}{0,013} \\
\hline & Femenino & 82 & 27 & 217 & 73 & 299 & 100 & \\
\hline \multirow{2}{*}{ Grupo poblacional } & Adolescentes & 4 & 19 & 17 & 81 & 21 & 100 & \multirow{2}{*}{0,330} \\
\hline & Adultos & 99 & 31 & 224 & 69 & 323 & 100 & \\
\hline \multirow{2}{*}{ Escolaridad } & Primaria y secundaria & 22 & 29 & 54 & 71 & 76 & 100 & \multirow{2}{*}{0,942} \\
\hline & Universitaria & 81 & 30 & 187 & 70 & 268 & 100 & \\
\hline \multirow{2}{*}{ Proveniencia de los ingresos } & Ingresos propios & 79 & 33 & 163 & 67 & 242 & 100 & \multirow{2}{*}{0,119} \\
\hline & Ingresos ajenos & 24 & 24 & 78 & 76 & 102 & 100 & \\
\hline \multirow{2}{*}{ Tener comorbilidades } & Sí & 25 & 30 & 59 & 70 & 84 & 100 & \multirow{2}{*}{0,966} \\
\hline & No & 78 & 30 & 182 & 70 & 260 & 100 & \\
\hline \multirow{2}{*}{ Antecedentes familiares de enfermedad celiaca } & Sí & 57 & 34 & 113 & 66 & 170 & 100 & \multirow{2}{*}{0,159} \\
\hline & No & 46 & 26 & 128 & 74 & 174 & 100 & \\
\hline \multirow{2}{*}{ Molestias al trasgredir la DSG } & Sí & 70 & 27 & 189 & 73 & 259 & 100 & \multirow{2}{*}{0,039} \\
\hline & No & 33 & 39 & 52 & 61 & 85 & 100 & \\
\hline
\end{tabular}

*Valores de p según la prueba exacta de Fischer. DSG= dieta sin gluten.

\section{DISCUSIÓN}

La adherencia a la DSG medida con el CDAT de Leffler fue del $78 \%$, la calidad de vida medida con el CDAT de Dorn fue buena en el $30 \%$ y mala en el $70 \%$ de los participantes en el estudio. Además, el porcentaje de sujetos con mala calidad de vida fue más elevado en aquellos sin adherencia a la DSG $(84,2 \%)$ en comparación con aquellos con adherencia moderada $(70,3 \%)$ o buena $(64,7 \%)$. En aquellos con mala calidad de vida, el promedio de edad y el promedio de años del diagnóstico de la enfermedad celiaca fueron menores en comparación con quienes tenían buena calidad de vida.
La mala adherencia a la DSG fue más frecuente en los celiacos con mala calidad de vida, así como lo describen diversos autores (16). Esta asociación podría explicarse por las limitaciones alimentarias y sociales que acarrean esta restricción alimentaria, aunque este tema debe explorarse en profundidad. En 2017, la buena calidad de vida de los celiacos en Paraguay era del $25 \%$ y, según la presente encuesta, ha subido al $30 \%$, usando el mismo instrumento de medición (4). Esta diferencia podría deberse al mayor tamaño de la muestra del actual estudio, a la inclusión de adolescentes y a otros factores circunstanciales. 
Definir la calidad de vida es muy difícil porque depende de las expectativas y actitudes de cada persona. Aun así, los celiacos sufren situaciones comunes ocasionadas por sus restricciones dietéticas que les obligan a modificar su estilo de vida (17). Los factores demográficos, culturales, económicos y sociales afectan a cada individuo de diferente manera. Es más, pueden ir cambiando por región y hasta en el tiempo. En Eslovenia, utilizando el cuestionario EQ-5D, se detectó la adherencia a la DSG en el $82 \%$ de los celiacos encuestados. La buena calidad de vida se asoció con la duración prolongada de la dieta ( $15 \pm 6$ años) y al elevado nivel educativo. Por el contrario, la mala calidad de vida se observó en los sujetos mayores y con más comorbilidades (18). En Polonia, aplicando el cuestionario HRQOL, la buena adherencia se observó en el $83 \%$ de los celiacos, predominó en aquellos con escaso nivel económico y afectó significativamente su calidad de vida. La formación académica, el lugar de residencia y la duración de la DSG no influenciaron la adherencia dietética (19). En Chile, utilizando encuestas telefónicas de percepción de adherencia a DSG, se halló que esta era estricta en el $70 \%$ de los niños celiacos, pero no se evaluó la calidad de vida (20). Si bien estos reportes citados utilizaron cuestionarios diferentes al CDAT de Leffler, pueden servir de aproximación a la situación de la adherencia a la DSG hallada en esta muestra en comparación con otros países.

A pesar de la presencia del gluten en el $70 \%$ de los alimentos industrializados, revisiones sistemáticas reportan que la adherencia a la DSG se logra entre el 45 y el $80 \%$ de los celiacos (2). En el presente estudio, la adherencia se encontró en un $78 \%$. Una investigación identificó diversos factores facilitadores significativos para realizar la DSG: aumento de la educación (22,5\%), mayor conocimiento de la dieta (20\%), mayor voluntad y autocontrol $(17,5 \%)$ y pertenencia a asociaciones de celiacos (12,5\%). Las barreras más importantes fueron menor conocimiento de la enfermedad celiaca (35\%), compras en restaurantes y supermercados (30\%), mala educación del paciente por parte del médico (17,5\%), baja intención y motivación para adherirse a una DSG (17,5\%) (11). Todos estos factores quedan pendientes de estudio en Paraguay.

En este estudio no se halló diferencia en la frecuencia de los tipos de presentación clínica de la enfermedad celiaca y la calidad de vida, a diferencia de los datos obtenidos por Kurppa et al. (16), quienes demostraron que la DSG alivia sustancialmente las molestias gastrointestinales y mejoran la calidad de vida en comparación con la referida antes del diagnóstico (16). Esto es comprensible porque la diarrea crónica, la dispepsia y el meteorismo son molestos para los pacientes y todos mejoran con la DSG (1). Pero también es cierto que las restricciones dietéticas de los celiacos implican mayores gastos, distanciamiento social, dificultades para los viajes y depresión. Posiblemente alguno de estos factores justifique que los pacientes encuestados no hayan mejorado su calidad de vida.

En la niñez, es más fácil para los padres controlar la calidad de los alimentos ingeridos dentro del hogar (15); pero en la adolescencia ese control disminuye sustancialmente y prima mucho la presión social de las amistades, todo lo cual disminuye la adherencia a la DSG y altera la calidad de vida en ese grupo etario, como se halló en esta muestra (16). Tal hallazgo concuerda con otros estudios en los que la hipervigilancia de los familiares generó frustración y ansiedad en los celiacos (12). Por lo tanto, se recomienda mejorar la educación de los adolescentes para que al inicio de su vida independiente sean más responsables al momento de elegir sus alimentos. En este sentido, es de gran utilidad la función de los grupos de autoayuda. La 
participación en las asociaciones de celiacos ha demostrado que favorece la adherencia a la DSG. Ello se debe a que en esos colectivos adquieren información certera sobre el manejo de su afección, reciben contención emocional y aprenden a cocinar sus propios alimentos sin riesgo de contaminación con gluten (21).

La frecuencia de mala calidad de vida predominó en las mujeres. Ello podría deberse a que la enfermedad celiaca en este sexo es más frecuente (21). Otra posibilidad es porque se ha demostrado que las mujeres tienden a ser más exigentes, por lo cual su calidad de vida también se ve más afectada (16). Este fenómeno amerita una investigación de carácter cualitativo.

Según la literatura, la presencia de comorbilidades se asocia con mala calidad de vida, pero en esta muestra no se encontró ese fenómeno. Se estima que los celiacos con otras enfermedades requieren más exigencias para su control, como la insulinoterapia. En este estudio llamó la atención la presencia de hipotiroidismo primario como la principal comorbilidad. La asociación entre la tiroiditis crónica autoinmune y la enfermedad celiaca fue demostrada en Paraguay, donde el $13 \%$ de los afectados con hipotiroidismo eran portadores de enfermedad celiaca asintomática (22). Por ello el tamizaje de esta alteración hormonal es recomendable al momento del diagnóstico de la enfermedad celiaca.

En este estudio, los celiacos con mala calidad de vida dependen de los ingresos financieros ajenos en un $75 \%$. Algunos reportes han demostrado que el nivel socioeconómico bajo y la falta de ingresos propios dificultan el acceso a la DSG y empeoran la calidad de vida $(19,21)$. Un factor que puede influir en ese aspecto es el elevado costo de los alimentos industrializados sin gluten. Si bien en el Paraguay se dispone con facilidad de alimentos naturalmente libres de gluten a base de cereales (maíz, arroz) y féculas (mandioca o yuca), cuando estos son industrializados elevan considerablemente sus costos en comparación con aquellos con gluten. Esta diferencia de precios se ve en todos los países, aunque en Paraguay aún no ha sido cuantificado (23).

La ingesta de gluten, accidental o voluntaria, generó molestias digestivas al $46 \%$ de los celiacos de esta muestra. Diversos estudios han demostrado que la ingesta continua de gluten está presente en el $30 \%$ de los celiacos. Aunque la misma no genere síntomas, perpetúa la inflamación de la mucosa intestinal, la persistencia de los anticuerpos y predispone a la aparición de complicaciones crónicas (24). Se asocia, además, con una mala calidad de vida (25). Por ello es indispensable que los celiacos conozcan en profundidad los detalles de su condición y sean monitoreados en su tratamiento nutricional. En aquellos con mala adherencia a la DSG detectados en el presente estudio, se impone una investigación con diseño cualitativo para conocer las causas de las trasgresiones y tomar medidas.

Las limitaciones de este estudio son varias. En primer lugar, no se puede tener la certeza de que todos los encuestados tenían confirmada la enfermedad celiaca. No se ha realizado una adaptación transcultural de los instrumentos de medición. No se dispone de un parámetro comparativo de la calidad de vida en la población general. No se utilizó un diseño longitudinal que pudiera obtener datos antes y después de detectarse la enfermedad celiaca (18). Tampoco puede descartarse el efecto de la pandemia por SARS-CoV-2 vigente durante el estudio. La enfermedad celiaca no es una afección que predisponga a las infecciones como el SARS-CoV-2 (26). El impacto en el aislamiento generado por la pandemia no ha generado cambios en la DSG en $69 \%$ de los adultos y en 


\section{Dieta sin gluten y calidad de vida}

$70 \%$ de los niños de Italia, y mejoró para el $29 \%$ de ambos (27). En la muestra del presente estudio no se puede saber ese impacto, pues no se tienen datos previos. La pandemia ha generado ansiedad, depresión e insomnio en la población paraguaya (28). Así mismo, ha obligado a muchos individuos a permanecer en sus casas, lo que les permitiría realizar su DSG (26). Sería importante volver a aplicar la misma encuesta una vez finalizada esta pandemia, incluyendo también a niños y a sus familiares. Otro aspecto que debería explorarse es el efecto de las creencias religiosas en la calidad de vida, pues es sabido que los católicos deben recibir la comunión con hostias hechas de trigo (21).

Las fortalezas fueron el tamaño adecuado de la muestra y la inclusión de celiacos de todas las regiones del Paraguay (12). Es el primer estudio del país que aporta información relevante sobre los factores que alteran la calidad de vida y pueden ser modificados. En este aspecto, la Fundación Paraguaya de Celiacos (www.fupacel.org) juega un rol determinante, ya que se ha fijado como meta mejorar la calidad de vida de los celiacos.

En conclusión, la escasa adherencia a la dieta sin gluten observada en esta muestra es más frecuente en los celiacos con menor calidad de vida asociada con la salud. Se sugieren estudios que identifiquen los principales factores que puedan constituir las barreras para lograr mejorar la calidad de vida de los celiacos.

\section{CONFLICTOS DE INTERESES}

Los autores declaran no presentar conflictos de intereses en esta investigación.

\section{Contribución de los autores}

Todos los autores contribuyeron igualmente en el manuscrito.

\section{Financiación}

Autofinanciado por los autores.

\section{- -Referencias}

1. Al-Toma A, Volta U, Auricchio R, Castillejo G, Sanders DS, Cellier C, et al. European Society for the Study of Coeliac Disease (ESsCD) guideline for coeliac disease and other gluten-related disorders. United Eur Gastroenterol J. 2019;7(5):583-613. https://doi.org/10.1177/2050640619844125

2. Canestaro WJ, Edwards TC, Patrick DL. Systematic review: patient-reported outcome measures in coeliac disease for regulatory submissions. Aliment Pharmacol Ther. 2016;44(4):313-31. https://doi.org/10.1111/apt.13703

3. Elli L, Ferretti F, Orlando S, Vecchi M, Monguzzi E, Roncoroni L, et al. Management of celiac disease in daily clinical practice. Eur J Intern Med. 2019;61:15-24. https://doi.org/10.1016/j.ejim.2018.11.012

4. Real-Delor RE, Centurión-Medina IC. Calidad de vida en adultos del Paraguay con enfermedad celiaca. Duazary. 2018;15(1):61-70. https://doi.org/10.21676/2389783X.2026

5. Casellas F, Rodrigo L, Lucendo AJ, Fernández-Bañares F, Molina-Infante J, Vivas S, et al. Benefit on health-related quality of life of adherence to gluten-free diet in adult patients with celiac disease. Rev Esp Enferm Dig. 2015;107(4):196-201. Disponible en: https://scielo.isciii.es/scielo.php?script=sci_arttext\&pid=S1130-01082015000400002

6. White LE, Bannerman E, Gillett PM. Coeliac disease and the gluten-free diet: A review of the burdens; factors associated with adherence and impact on health-related quality of life, with specific focus on adolescence. J Hum Nutr Diet. 2016;29(5):593-606. https://doi.org/10.1111/jhn.12375 
7. Dorn SD, Hernandez L, Minaya MT, Morris CB, Hu Y, Leserman J, et al. The development and validation of a new coeliac disease quality of life survey (CD-QOL). Aliment Pharmacol Ther. 2010 Mar;31(6):666-75. https://doi.org/10.1111/j.1365-2036.2009.04220.x

8. Fueyo-Díaz R, Montoro M, Magallón-Botaya R, Gascón-Santos S, Asensio-Martínez Á, Palacios-Navarro G, et al. Influence of compliance to diet and self-efficacy expectation on quality of life in patients with celiac disease in Spain. Nutrients. 2020 Sep 2;12(9):2672. https://doi.org/10.3390/nu12092672

9. Rodrigo L, Pérez-Martinez I, Lauret-Braña E, Suárez-González A. Descriptive study of the different tools used to evaluate the adherence to a gluten-free diet in celiac disease patients. Nutrients. 2018;10(11):1777. https://doi.org/10.3390/nu10111777

10. Fueyo-Díaz R, Gascón-Santos S, Asensio-Martínez Á, Sánchez-Calavera MA, Magallón-Botaya R. Transcultural adaptation and validation of the celiac dietary adherence test. A simple questionnaire to measure adherence to a gluten-free diet. Rev Esp Enferm Dig. 2016;108(3):138-44. https://doi.org/10.17235/reed.2016.4033/2015

11. Abu-Janb N, Jaana M. Facilitators and barriers to adherence to gluten-free diet among adults with celiac disease: A systematic review. J Hum Nutr Diet. 2020;36(6):786-810. https://doi.org/10.1111/jhn.12754

12. Wolf RL, Lebwohl B, Lee AR, Zybert P, Reilly NR, Cadenhead J, et al. Hypervigilance to a gluten-free diet and decreased quality of life in teenagers and adults with celiac disease. Dig Dis Sci. 2018;63(6):1438-48. https://doi.org/10.1007/s10620-018-4936-4

13. Leffler DA, Dennis M, Edwards-George JB, Jamma S, Magge S, Cook EF, et al. A simple validated gluten-free diet adherence survey for adults with celiac disease. Clin Gastroenterol Hepatol. 2009;7(5):530-6. https://doi.org/10.1016/j.cgh.2008.12.032

14. Nikniaz Z, Asghari-Jafarabadi M, Ghaffarifar S, Saeedi Z, Akbari-Namvar Z, Shirmohammadi M. Psychometric properties of the Persian version of the celiac disease adherence test questionnaire. BMC Gastroenterol. 2020;20(1):247. https://doi.org/10.1186/s12876-020-01396-8

15. Johansson K, Norström F, Nordyke K, Myleus A. Celiac dietary adherence test simplifies determining adherence to a gluten-free diet in Swedish adolescents. J Pediatr Gastroenterol Nutr. 2019;69(5):575-80. https://doi.org/10.1097/MPG.0000000000002451

16. Kurppa K, Collin P, Mäki M, Kaukinen K. Celiac disease and health-related quality of life. Expert Rev Gastroenterol Hepatol. 2011;5(1):83-90. https://doi.org/10.1586/egh.10.81

17. Santolaria-Piedrafita S, Montoro-Huguet M. Celiac disease, gluten-free diet and health-related quality of life. Rev Esp Enferm Dig. 2015;107(4):193-5. Disponible en: https://pubmed.ncbi.nlm.nih.gov/25824916/

18. Turk E, Mičetić-Turk D, Šikić-Pogačar M, Tapajner A, Vlaisavljević V, Prevolnik-Rupel V. Health related QoL in celiac disease patients in Slovenia. Heal Qual Life Outcomes. 2020;18(1):356. https://doi.org/10.1186/s12955-020-01612-9

19. Zysk W, Głąbska D, Guzek D. Social and emotional fears and worries influencing the quality of life of female celiac disease patients following a gluten-free diet. Nutrients. 2018;10(10):1414. https://doi.org/10.3390/nu10101414

20. Espino A, Castillo LC, Guiraldes E, Santibáñez H, Miquel JF, Santibanez H, et al. Encuesta nacional online aplicada en pacientes con enfermedad celíaca en Chile. Rev Med Chil. 2011;139(7):841-7. https://doi.org/10.4067/S0034-98872011000700003

21. Silva LA da, Bessa CC, Guedes NG, Lopes MV de O, Silva VM da, Santos JC Dos, et al. Accuracy of the clinical indicators of ineffective health management in celiac people. Rev Bras Enferm. 2020;73(3):e20180739. https://doi.org/10.1590/0034-7167-2018-0739

22. Real RE, Valenzuela JA, González NR. Enfermedad celiaca silente en pacientes adultos con enfermedades tiroideas autoinmunes. An Fac Cienc Méd. 2020;53(1):71-80. https://doi.org/10.18004/anales/2020.053.01.71-080 


\section{Dieta sin gluten y calidad de vida}

23. Lee AR, Wolf RL, Lebwohl B, Ciaccio EJ, Green PHR. Persistent economic burden of the gluten free diet. Nutrients. 2019;11(2):399. https://doi.org/10.3390/nu11020399

24. Kaukinen K. Updates on systemic consequences of coeliac disease. Nat Rev Gastroenterol Hepatol. 2021;18(2):87-8. https://doi.org/10.1038/s41575-020-00387-0

25. Elli L, Bascuñán K, Di Lernia L, Bardella MT, Doneda L, Soldati L, et al. Safety of occasional ingestion of gluten in patients with celiac disease: A real-life study. BMC Med. 2020;18(1):42. https://doi.org/10.1186/s12916-020-1511-6

26. Elli L, Barisani D, Vaira V, Bardella MT, Topa M, Vecchi M, et al. How to manage celiac disease and gluten-free diet during the COVID-19 era: proposals from a tertiary referral center in a high-incidence scenario. BMC Gastroenterol. 2020;20(1):387. https://doi.org/10.1186/s12876-020-01524-4

27. Monzani A, Lionetti E, Felici E, Fransos L, Azzolina D, Rabbone I, et al. Adherence to the gluten-free diet during the lockdown for covid-19 pandemic: A web-based survey of italian subjects with celiac disease. Nutrients. 2020;12(11):3467. https://doi.org/10.3390/nu12113467

28. Ferreira MS, Coronel G, Rivarola MA. Impacto sobre la salud mental durante la pandemia COVID 19 en Paraguay. Rev virtual Soc Parag Med Int. 2021;8(1):61-8. https://doi.org/10.18004/rvspmi/2312-3893/2021.08.01.61 